Kragujevac Journal of Mathematics

Volume 42(2) (2018), Pages 249-258.

\title{
SERVAL ALGEBRAIC IDENTITIES IN 3-PRIME NEAR-RINGS
}

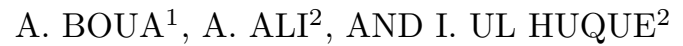

\begin{abstract}
The purpose of this paper is to study derivations and generalized derivations satisfying certain differential identities on Jordan ideals and Lie ideals of 3prime near-rings. Moreover, we provide examples to show that hypothesis of our results are necessary.
\end{abstract}

\section{INTRODUCTION}

Throughout this paper, $\mathcal{N}$ will be a left near-ring with multiplicative center $Z(\mathcal{N})$; and usually $\mathcal{N}$ will be 3-prime if for all $x, y \in \mathcal{N}, x \mathcal{N} y=\{0\}$ implies $x=0$ or $y=0$. A near-ring $\mathcal{N}$ is called zero-symmetric if $0 x=0$, for all $x \in \mathcal{N}$ (recall that left distributivity yields $x 0=0$ ). According to the reference [12], an abelian near-ring $\mathcal{N}$ is a near-ring such that $(\mathcal{N},+)$ is abelian. An additive mapping $d: \mathcal{N} \rightarrow \mathcal{N}$ is a derivation if $d(x y)=x d(y)+d(x) y$, for all $x, y \in \mathcal{N}$, or equivalently, as noted in [13], that $d(x y)=d(x) y+x d(y)$, for all $x, y \in \mathcal{N}$. Let $d$ be a derivation of $\mathcal{N}$, an additive mapping $F: \mathcal{N} \rightarrow \mathcal{N}$ is said to be a right generalized derivation of $\mathcal{N}$ associated with $d$ if $F(x y)=F(x) y+x d(y)$, for all $x, y \in \mathcal{N}$ and $F$ is said to be a left generalized derivation of $\mathcal{N}$ associated with $d$ if $F(x y)=d(x) y+x F(y)$, for all $x, y \in \mathcal{N}$. Moreover, $F$ is said to be a generalized derivation of $\mathcal{N}$ associated with $d$ if it is both a right and left generalized derivation of $\mathcal{N}$ associated with $d$. Note that, in 3-prime near-ring, $F=0$ implies that $d=0$. For any pair of elements $x, y \in \mathcal{N}$, $[x, y]=x y-y x$ and $x \circ y=x y+y x$ will denote the well-known Lie product and Jordan product respectively. Recall that $\mathcal{N}$ is called 2-torsion free if $2 x=0$ implies $x=0$, for all $x \in \mathcal{N}$. An additive subgroup $J$ of $\mathcal{N}$ is said to be Jordan left (resp. right) ideal of $\mathcal{N}$ if $n \circ j \in J$ (resp. $j \circ n \in J$ ), for all $j \in J, n \in \mathcal{N}$ and $J$ is said to be a Jordan ideal of $\mathcal{N}$ if $j \circ n \in J$ and $n \circ j \in J$, for all $j \in J, n \in \mathcal{N}$. An additive

Key words and phrases. 3-prime near-rings, derivations, Jordan ideals, Lie ideals.

2010 Mathematics Subject Classification. Primary: 16N60. Secondary: 16W25, 16 Y30.

Received: May 19, 2016.

Accepted: March 06, 2017. 
subgroup $U$ of $\mathcal{N}$ is said to be Lie ideal of $\mathcal{N}$ if $[u, n] \in U$, for all $u \in U, n \in \mathcal{N}$. In $[1$, $5,6,10]$ and [13] commutativity of near ring satisfying algebraic conditions involving derivations been studied. In the present paper, we continue the line of investigation regarding the study of commutativity of Jordan ideal and Lie ideal of a 3-prime near ring admitting a nonzero derivation satisfying some algebraic identities.

\section{Some Preliminaries}

We begin with the following results which will be used extensively to prove our theorem. The first Lemma appears in [4], [5] and [13].

Lemma 2.1. Let $\mathcal{N}$ be a 3-prime near-ring and $d$ a nonzero derivation of $\mathcal{N}$.

(i) If $z \in Z(\mathcal{N}) \backslash\{0\}$ and $x z \in Z(\mathcal{N})$, then $x \in Z(\mathcal{N})$.

(ii) If $x \in \mathcal{N}$ and $x d(\mathcal{N})=\{0\}$, then $x=0$.

(iii) If $\mathcal{N}$ is 2-torsion free, then $d^{2} \neq 0$.

(iv) If $x \in Z(\mathcal{N})$, then $d(x) \in Z(\mathcal{N})$.

Lemma 2.2. [4, Lemma 1] Let $\mathcal{N}$ be a near-ring and $d$ be a derivation of $\mathcal{N}$. Then $\mathcal{N}$ satisfies the following partial distributive law

$$
(x d(y)+d(x) y) z=x d(y) z+d(x) y z, \quad \text { for all } x, y, z \in \mathcal{N} .
$$

Lemma 2.3. Let $d$ be an arbitrary additive endomorphism of $\mathcal{N}$. Then $d(x y)=$ $x d(y)+d(x) y$, for all $x, y \in \mathcal{N}$ if and only if $d(x y)=d(x) y+x d(y)$, for all $x, y \in \mathcal{N}$. Therefore $d$ is a derivation if and only if $d(x y)=d(x) y+x d(y)$, for all $x, y \in \mathcal{N}$.

Recall that a map $d: \mathcal{N} \rightarrow \mathcal{N}$ is called a multiplicative derivation on $N$ if $d(x y)=$ $x d(y)+d(x) y$, for all $x, y \in \mathcal{N}$. Notice that any derivation on $\mathcal{N}$ is a multiplicative derivation.

Lemma 2.4. [11, Lemma 2.1] A near-ring $\mathcal{N}$ admits a multiplicative derivation if and only if it is zero-symmetric.

Using Lemma 2.4, we deduce that in all our results in the paper that $\mathcal{N}$ is a zero-symmetric near-ring.

Lemma 2.5. [9, Corollary 3] Let $\mathcal{N}$ be a 2-torsion free 3-prime near-ring and $J$ be a nonzero Jordan ideal of $\mathcal{N}$. If $\mathcal{N}$ admits a derivation $d$ such that $d(J)=\{0\}$, then $d=0$ or the element of $J$ commute under the multiplication of $\mathcal{N}$.

Lemma 2.6. Let $\mathcal{N}$ be a 3-prime near-ring and $J$ a nonzero Jordan ideal of $\mathcal{N}$. If the element of $J$ commute under the multiplication of $\mathcal{N}$, then $J$ is commutative.

Proof. Using the same proof in [8, Remark 1], we find the required result.

Lemma 2.7. [8, Lemma 2 and Lemma 3] Let $\mathcal{N}$ be a 2-torsion free 3-prime near-ring and $J$ a nonzero Jordan ideal of $\mathcal{N}$.

(i) If $j^{2}=0$, for all $j \in J$, then $J=\{0\}$. 
(ii) If $J \subseteq Z(\mathcal{N})$, then $\mathcal{N}$ is a commutative ring.

Lemma 2.8. Let $\mathcal{N}$ be a 3-prime near ring and $U$ be a nonzero Lie ideal of $\mathcal{N}$.

(i) If $x U=\{0\}$, for all $x \in \mathcal{N}$, then $x=0$.

(ii) If $U \subseteq Z(\mathcal{N})$, then $(\mathcal{N},+)$ is abelian.

Proof. (i) Suppose that $x U=\{0\}$. Then $x[u, n]=0$, for all $u \in U$ and $n \in \mathcal{N}$ which implies that $x \mathcal{N} u=\{0\}$, for all $u \in U$. By 3-primeness of $\mathcal{N}$, we have $x=0$.

(ii) For all $x, y \in \mathcal{N}$ and $u \in U$, we have

$$
\begin{aligned}
(x+y)(u+u) & =(u+u)(x+y), \\
(x+y) u+(x+y) u & =(u+u) x+(u+u) y, \\
u(x+y)+u(x+y) & =x(u+u)+y(u+u), \\
u x+u y+u x+u y & =x u+x u+y u+y u, \\
u x+u y+u x+u y & =u x+u x+u y+u y .
\end{aligned}
$$

This implies that $u(x+y-x-y)=0$. Since $U \subseteq Z(\mathcal{N})$, then by part (i), we get $x+y=y+x$, for all $x, y \in \mathcal{N}$ which completes the proof.

\section{Main Results}

In this section, we give some new results and examples concerning the existence of Jordan ideal and derivations in near-rings. We begin this section by the following interesting results for near-rings.

Theorem 3.1. Let $\mathcal{N}$ be a 2-torsion free 3-prime near-ring and $J$ a nonzero right Jordan ideal of $\mathcal{N}$ such that $J \cap Z(\mathcal{N})-\{0\} \neq \emptyset$. If $\mathcal{N}$ admits a nonzero derivation $d$ for which $d^{2}(J)=\{0\}$, then $J$ is commutative.

Proof. Let $d^{2}(j)=0$, for all $j \in J$. Replacing $j$ by $j \circ n$ for all $n \in \mathcal{N}$ and using the definition of $d$ together with our initial hypothesis, we obtain for all $j \in J, n \in \mathcal{N}$

$$
2 d(j) d(n)+j d^{2}(n)+d^{2}(n) j+2 d(n) d(j)=0 \quad \text { for all } j \in J, n \in \mathcal{N} .
$$

Applying $d$ to (3.1) gives for all $j \in J, n \in \mathcal{N}$

$$
2 d(j) d^{2}(n)+d(j) d^{2}(n)+j d^{3}(n)+d^{3}(n) j+d^{2}(n) d(j)+2 d^{2}(n) d(j)=0 .
$$

Taking $d(n)$ in place of $n$ in (3.1) yields

$$
2 d(j) d^{2}(n)+j d^{3}(n)+d^{3}(n) j+2 d^{2}(n) d(j)=0 \quad \text { for all } j \in J, n \in \mathcal{N} .
$$

Using (3.3) in (3.2), we get

$$
d(j) d^{2}(n)+d^{2}(n) d(j)=0, \quad \text { for all } j \in J, n \in \mathcal{N} .
$$

For $z \in Z(\mathcal{N})$, replacing $n$ by $z$ in (3.4), it follows from (3.4) that $2 d^{2}(z) d(j)=0$, for all $j \in J$. By 2 -torsion freeness with Lemma 2.1 (iv), we obtain $d^{2}(z) \mathcal{N} d(J)=\{0\}$, for all $z \in Z(\mathcal{N})$. The 3-primeness of $\mathcal{N}$ implies that

$$
d^{2}(z)=0 \text { or } d(j)=0, \quad \text { for all } j \in J, z \in Z(\mathcal{N}) .
$$


If $d(j)=0$, for all $j \in J$, then by Lemma 2.5 and Lemma 2.6, we find that $J$ is commutative.

If $d^{2}(z)=0$ for all $z \in Z(\mathcal{N})$. Replacing $z$ by $y z$ where $y \in Z(\mathcal{N})$, we find that $d^{2}(y) z+2 d(y) d(z)+y d^{2}(z)=0$, for all $y, z \in Z(\mathcal{N})$ which reduces to $2 d(y) d(z)=0$, for all $y, z \in Z(\mathcal{N})$. In view of the 2-torsion freeness and 3 -primeness of $\mathcal{N}$, we arrive at $d(Z(\mathcal{N}))=\{0\}$ and by hypothesis, there exists $j_{0} \in J$ and $j_{0} \in Z(\mathcal{N})-\{0\}$ such that

$$
\begin{aligned}
0 & =d^{2}\left(n \circ j_{0}\right) \\
& =d^{2}\left(n\left(j_{0}+j_{0}\right)\right) \\
& =d^{2}(n)\left(j_{0}+j_{0}\right), \quad \text { for all } n \in \mathcal{N} .
\end{aligned}
$$

This reduces to $d^{2}(n) j_{0}=0$, for all $j_{0} \in J$, so $d^{2}(n) \mathcal{N} j_{0}=\{0\}$, for all $n \in \mathcal{N}$. Since $\mathcal{N}$ is 3 -prime and $j_{0} \neq 0$, we have $d^{2}=0$, a contradiction with Lemma 2.1 (iii).

Theorem 3.2. Let $\mathcal{N}$ be a 2-torsion free 3-prime near-ring and $J$ be a nonzero right Jordan ideal of $\mathcal{N}$. If $\mathcal{N}$ admits a nonzero derivation $d$, then the following assertions are equivalent.

(i) $d(j n) \in Z(\mathcal{N})$, for all $j \in J, n \in \mathcal{N}$.

(ii) $\mathcal{N}$ is a commutative ring.

Proof. It is obvious that (ii) implies (i). So we need to prove that (i) $\Rightarrow$ (ii).

(i) $\Rightarrow$ (ii) Suppose that $d(j n) \in Z(\mathcal{N})$, for all $j \in J, n \in \mathcal{N}$. This implies that $d(j) n+j d(n) \in Z(\mathcal{N})$, for all $j \in J, n \in \mathcal{N}$. Replacing $n$ by $i n$, we get

$$
(d(j) i n+j d(i n)) j=j(d(j) i n+j d(i n)), \quad \text { for all } i, j \in J, n \in \mathcal{N} .
$$

Using our hypothesis, the above relation gives

$$
d(j) i n j=j d(j) i n, \quad \text { for all } i, j \in J, n \in \mathcal{N} .
$$

Taking $m n$ instead of $n$ in the last equation and using it again, we arrive at

$$
d(j) i \mathcal{N}[j, n]=\{0\}, \quad \text { for all } i, j \in J, n \in \mathcal{N} .
$$

By 3-primeness of $\mathcal{N}$, we find that

$$
d(j) i=0 \text { or } j \in Z(\mathcal{N}) \text { for all } i, j \in J .
$$

If there exists $j_{0} \in J$ such that $d\left(j_{0}\right) i=0$, for all $i \in J$, then replacing $i$ by $i \circ n$, we have

$$
\begin{aligned}
0 & =d\left(j_{0}\right)(i n+n i) \\
& =d\left(j_{0}\right) i n+d\left(j_{0}\right) n i \\
& =d\left(j_{0}\right) n i, \quad \text { for all } i \in J, n \in \mathcal{N}
\end{aligned}
$$

this expression reduced to $d\left(j_{0}\right) \mathcal{N} i=\{0\}$, for all $i \in J$. Since $J \neq\{0\}$, then 3 primeness of $\mathcal{N}$ forces that $d\left(j_{0}\right)=0$. In this case (3.6) becomes

$$
d(j)=0 \text { or } j \in Z(\mathcal{N}) \text { for all } j \in J .
$$


If there exists $j_{0} \in J$ such that $d\left(j_{0}\right)=0$, then by hypothesis we have $j_{0} d(n) \in Z(\mathcal{N})$, for all $n \in \mathcal{N}$. Replacing $n$ by $n m$, we get $j_{0} d(n) m+j_{0} n d(m) \in Z(\mathcal{N})$, for all $m, n \in \mathcal{N}$ which implies that

$$
m\left(j_{0} d(n) m+j_{0} n d(m)\right)=\left(j_{0} d(n) m+j_{0} n d(m)\right) m \text { for all } m, n \in \mathcal{N} .
$$

By a simple calculation, (3.8) can be rewritten as

$$
m j_{0} n d(m)=j_{0} n d(m) m, \text { for all } m, n \in \mathcal{N} \text {. }
$$

Replacing $n$ by $n j_{0}$ in the last expression and using it again, we arrive at

$$
j_{0} d(m) \mathcal{N}\left(m j_{0} n-j_{0} n m\right)=\{0\} \quad \text { for all } m, n \in \mathcal{N} .
$$

Using again the 3-primeness of $\mathcal{N}$, (3.9) gives

$$
j_{0} d(m)=0 \text { or } m j_{0} n=j_{0} n m \text { for all } m, n \in \mathcal{N} .
$$

Suppose there exists $m_{0} \in \mathcal{N}$ such that $m_{0} j_{0} n=j_{0} n m_{0}$, for all $n \in \mathcal{N}$. Taking $n t$ instead of $n$ in the last equation and invoking it again, we arrive at $j_{0} \mathcal{N}\left[m_{0}, t\right]=\{0\}$, for all $t \in \mathcal{N}$ and by application the 3-primeness of $\mathcal{N}$, we obtain $j_{0}=0$ or $m_{0} \in Z(\mathcal{N})$. In this case (3.10) becomes

$$
j_{0} d(m)=0 \text { or } m \in Z(\mathcal{N}) \text { for all } m \in \mathcal{N} .
$$

Suppose there exists $m_{0} \in Z(\mathcal{N})$. By hypothesis we have $j_{0} d\left(m_{0}\right) \in Z(\mathcal{N})$, using Lemma 2.1 (iv) and Lemma 2.1 (i), then either $d\left(m_{0}\right)=0$ or $j_{0} \in Z(\mathcal{N})$, then $(3.11)$ becomes

$$
j_{0} d(m)=0 \text { or } j_{0} \in Z(\mathcal{N}) \text { for all } m \in \mathcal{N} .
$$

Using the last expression with (3.10), we obtain

$$
j d(m)=0 \text { or } j \in Z(\mathcal{N}) \text { for all } j \in J, m \in \mathcal{N} .
$$

By Lemma 2.1(ii), we arrive at $j \in Z(\mathcal{N})$, for all $j \in J$ and Lemma 2.7 (ii) forces that $\mathcal{N}$ is a commutative ring.

Theorem 3.3. Let $\mathcal{N}$ be a 2 -torsion free 3-prime near-ring and $J$ a right Jordan ideal of $\mathcal{N}$. If $J$ has one of the following properties:

(i) $d(n \circ j)=[n, j]$, for all $j \in J, n \in \mathcal{N}$,

(ii) $d([n, j])=n \circ j$, for all $j \in J, n \in \mathcal{N}$,

(iii) $[d(n), j]=n \circ j$, for all $j \in J, n \in \mathcal{N}$,

(iv) $d(n) \circ j=[n, j]$, for all $j \in J, n \in \mathcal{N}$,

then $J$ is commutative.

Proof. (i) We are assuming that

$$
d(n \circ j)=[n, j], \quad \text { for all } j \in J, n \in \mathcal{N} .
$$


Replacing $n$ by $j n$ in (3.13) and using the fact that $[j n, j]=j[n, j]$ together with $j n \circ j=j(n \circ j)$, we have

$$
\begin{aligned}
j[n, j] & =[j n, j] \\
& =d(j n \circ j) \\
& =j d(n \circ j)+d(j)(n \circ j) \\
& =j[n, j]+d(j)(n \circ j), \quad \text { for all } j \in J, n \in \mathcal{N} .
\end{aligned}
$$

This expression gives us $d(j)(n \circ j)=0$, for all $j \in J, n \in \mathcal{N}$, it follows that

$$
d(j) n j=-d(j) j n, \quad \text { for all } j \in J, n \in \mathcal{N} .
$$

Substituting $n m$ in place of $n$ in (3.14), we get

$$
\begin{aligned}
d(j) n m j & =-d(j) j n m \\
& =d(j) j n(-m) \\
& =d(j) n(-j)(-m), \quad \text { for all } j \in J, m, n \in \mathcal{N},
\end{aligned}
$$

which can be rewritten as

$$
d(j) \mathcal{N}(-m(-j)+(-j) m)=\{0\}, \quad \text { for all } j \in J, m \in \mathcal{N} .
$$

Equivalently,

$$
d(-j) \mathcal{N}(-m j+j m)=\{0\}, \quad \text { for all } j \in J, m \in \mathcal{N} .
$$

By 3-primeness of $\mathcal{N}$, we have

$$
d(j)=0 \text { or } j \in Z(\mathcal{N}) \text { for all } j \in J .
$$

Suppose there is an element $j_{0} \in J$ such that $j_{0} \in Z(\mathcal{N})$. Then (3.13) becomes $2 d\left(n j_{0}\right)=0$, for all $n \in \mathcal{N}$ and using 2-torsion freeness of $\mathcal{N}$, we get $d\left(n j_{0}\right)=0$, for all $n \in \mathcal{N}$. Replacing $n$ by $n j_{0}$ in the last expression and using it with the definition of $d$, we arrive at $n \mathcal{N} j_{0} \mathcal{N} d\left(j_{0}\right)=\{0\}$. Again by 3-primeness of $\mathcal{N}$, we conclude that $d\left(j_{0}\right)=0$ in this case, (3.15) implies that $d(J)=\{0\}$ which forces that $J$ is commutative by Lemma 2.5 and Lemma 2.6.

(ii) Suppose that

$$
d([n, j])=n \circ j, \quad \text { for all } j \in J, n \in \mathcal{N} .
$$

Substituting $j n$ in place of $n$ in (3.16) and using the fact that $[j n, j]=j[n, j]$ together with $j n \circ j=j(n \circ j)$, we have

$$
\begin{aligned}
j(n \circ j) & =j n \circ j \\
& =d([j n, j]) \\
& =j d([n, j])+d(j)[n, j] \\
& =j(n \circ j)+d(j)[n, j], \quad \text { for all } j \in J, n \in \mathcal{N}
\end{aligned}
$$

which implies that $d(j)[n, j]=0$, for all $j \in J, n \in \mathcal{N}$. It follows that

$$
d(j) n j=d(j) j n, \quad \text { for all } j \in J, n \in \mathcal{N} .
$$


Replacing $n$ by $n m$ in the above equation and using it, we can easily get

$$
d(j) \mathcal{N}[m, j]=\{0\}, \quad \text { for all } j \in J, m \in \mathcal{N} .
$$

By 3-primeness of $\mathcal{N}$, we have

$$
d(j)=0 \text { or } j \in Z(\mathcal{N}) \text { for all } j \in J .
$$

If there is an element $j_{0} \in J$ such that $j_{0} \in Z(\mathcal{N})$, then (3.16) becomes $2 n j_{0}=0$, for all $n \in \mathcal{N}$. By the 2-torsion freeness of $\mathcal{N}$, we arrive at $n \mathcal{N} j_{0}=\{0\}$, for all $n \in \mathcal{N}$ and by 3-primeness of $\mathcal{N}$, we conclude that $j_{0}=0$. In this case $(3.18)$ forces that $d(j)=0$, for all $j \in J$ which shows that $J$ is commutative by Lemma 2.5 and Lemma 2.6.

(iii) Assume that

$$
[d(n), j]=n \circ j, \quad \text { for all } j \in J, n \in \mathcal{N} .
$$

Substituting $j n$ instead of $n$ in (3.19), we have $d(j n) j-j d(j n)=j(n \circ j)=j[d(n), j]$, for all $j \in J, n \in \mathcal{N}$. Using the definition of $d$ with Lemma 2.2, we obtain

(3.20) $j d(n) j+d(j) n j-j d(j) n-j^{2} d(n)=j d(n) j-j^{2} d(n) \quad$ for all $j \in J, n \in \mathcal{N}$.

Simplifying equation (3.20), we find that

$$
d(j) n j=j d(j) n, \quad \text { for all } j \in J, n \in \mathcal{N} .
$$

Using the same techniques as used after the equation (3.17), we can easily get the required result.

(iv) Assume that

$$
d(n) \circ j=[n, j] \text { for all } j \in J, n \in \mathcal{N} .
$$

Substituting $j n$ instead of $n$ in (3.22), we have $d(j n) j+j d(j n)=j[n, j]=j(d(n) \circ j)$, for all $j \in J, n \in \mathcal{N}$. Using the definition of $d$ and Lemma 2.2, we obtain

(3.23) $j d(n) j+d(j) n j+j d(j) n+j^{2} d(n)=j d(n) j+j^{2} d(n) \quad$ for all $j \in J, n \in \mathcal{N}$.

Simplifying equation (3.23), we find that

$$
d(j) n j=-j d(j) n, \quad \text { for all } j \in J, n \in \mathcal{N} .
$$

Applying the same techniques as used after the equation (3.17), we get the required result.

Theorem 3.4. Let $\mathcal{N}$ be a 2-torsion free 3-prime near-ring, $U$ a nonzero Lie ideal of $\mathcal{N}$ and $F$ a left generalized derivation associated with a derivation $d$. If $\mathcal{N}$ has one of the following properties:

(i) $F(u \circ n)=[u, n]$, for all $u \in U, n \in \mathcal{N}$,

(ii) $F([u, n])=u \circ n$, for all $u \in U, n \in \mathcal{N}$,

then $\mathcal{N}$ is abelian. 
Proof. (i) Suppose that

$$
F(u \circ n)=[u, n], \quad \text { for all } u \in U, n \in \mathcal{N} .
$$

Replacing $n$ by un in (3.25) and using it, we get

$$
\begin{aligned}
u F(u \circ n) & =u[u, n] \\
& =F(u(u \circ n)) \\
& =d(u)(u \circ n)+u F(u \circ n), \quad \text { for all } u \in U, n \in \mathcal{N} .
\end{aligned}
$$

This implies that

$$
d(u) u n=-d(u) n u, \quad \text { for all } u \in U, n \in \mathcal{N} .
$$

Since (3.26) is the same as (3.14), using the same techniques as used after the equation (3.14), we can easily arrive at

$$
d(u)=0 \text { or } u \in Z(\mathcal{N}) \text { for all } u \in U, n \in \mathcal{N} .
$$

If $d(u)=0$, for all $u \in U$, substituting $[u, n]$ in place of $u$ for $n \in \mathcal{N}$, we obtain $u d(n)=d(n) u$, for all $u \in U, n \in \mathcal{N}$. Now replacing $n$ by $d(n) m$ and using it, we have

$$
u d^{2}(n) m=d^{2}(n) m u, \quad \text { for all } u \in U m, n \in \mathcal{N} .
$$

Substituting $m r$ in place of $m$ in last expression and using it, we get $d^{2}(n) \mathcal{N}[u, r]=\{0\}$, for all $u \in U$ and $r, n \in \mathcal{N}$. By 3-primeness of $\mathcal{N}$, we have $u \in Z(\mathcal{N})$ or $d^{2}=0$. In this case Lemma 2.1 (iii) assures that $U \subseteq Z(\mathcal{N})$. Hence $\mathcal{N}$ is abelian by Lemma 2.8 (ii).

(ii) Suppose that

$$
F([u, n])=u \circ n, \quad \text { for all } u \in U, n \in \mathcal{N} .
$$

Replacing $n$ by un in (3.28) and using it, we get

$$
\begin{aligned}
u F([u, n]) & =u(u \circ n) \\
& =F(u[u, n]) \\
& =d(u)[u, n]+u F([u, n]), \quad \text { for all } u \in U, n \in \mathcal{N} .
\end{aligned}
$$

This implies that

$$
d(u) \text { un }=d(u) n u, \quad \text { for all } u \in U, n \in \mathcal{N} .
$$

Since (3.29) is the same as (3.17), using the same techniques as used after the equation (3.17), we can easily arrive at

$$
d(u)=0 \text { or } u \in Z(\mathcal{N}) \text { for all } u \in U, n \in \mathcal{N} .
$$

Arguing in the similar manner as we have done in part (i), we get the required result.

Theorem 3.5. Let $\mathcal{N}$ be a 2-torsion free 3-prime near-ring, $U$ a nonzero Lie ideal of $\mathcal{N}$ and $F$ a left generalized derivation associated with a derivation $d$ such that $F([u, n]) \pm(u \circ n) \in Z(\mathcal{N})$, for all $u \in U, n \in \mathcal{N}$, then $\mathcal{N}$ is abelian. 
Proof. Suppose that

$$
F([u, n])+(u \circ n) \in Z(\mathcal{N}) \text { for all } u \in U, n \in \mathcal{N} .
$$

Replacing $n$ by $u$, we obtain $u^{2} \in Z(\mathcal{N})$, for all $u \in U$. Putting $u^{2}$ in place of $n$ in (3.31), we get $u \circ u^{2} \in Z(\mathcal{N})$, for all $u \in U$ which implies that $u^{2}(2 u) \in Z(\mathcal{N})$, for all $u \in U$.

Since $u^{2} \in Z(\mathcal{N})$, for all $u \in \mathcal{U}$, then the last expression becomes

$$
u^{2}=0 \text { or } 2 u \in Z(\mathcal{N}), \text { for all } u \in U \text {. }
$$

If there is $u_{0} \in U$ such that $2 u_{0} \in Z(\mathcal{N})$, then replacing $n$ by $2 u_{0}$ in (3.32), we obtain $\left(2 u_{0}\right)(2 v) \in Z(\mathcal{N})$, for all $v \in U$ which forces that $2 u_{0}=0$ or $2 v \in Z(\mathcal{N})$, for all $v \in U$. By 2-torsion freeness of $\mathcal{N}$, we get

$$
u_{0}=0 \text { or } 2 v \in Z(\mathcal{N}), \quad \text { for all } v \in U .
$$

In this case, (3.32) becomes

$$
u^{2}=0 \text { or } 2 v \in Z(\mathcal{N}), \quad \text { for all } u, v \in U .
$$

If $2 v \in Z(\mathcal{N})$, for all $v \in U$, by (3.31), we have $2 v^{2} \in Z(\mathcal{N})$, for all $v \in U$. This implies that

$$
2 v=0 \text { or } v \in Z(\mathcal{N}), \text { for all } v \in U .
$$

By 2-torsion freeness of $\mathcal{N}$, we obtain $v=0$ or $v \in Z(\mathcal{N})$, for all $v \in U$ which implies that $U \subseteq Z(\mathcal{N})$. Hence $\mathcal{N}$ is abelian by Lemma 2.8 (ii).

The following example demonstrates that in Theorem 3.2 and Theorem 3.3 the restriction $\mathcal{N}$ to be 3 -prime near ring is not superfluous.

Example 3.1. Let $\mathcal{S}$ be a 2-torsion free left near-ring which is not abelian. Define $\mathcal{N}, J, d$ by

$$
\mathcal{N}=\left\{\left(\begin{array}{ccc}
0 & x & y \\
0 & 0 & 0 \\
0 & z & 0
\end{array}\right) \mid x, y, z \in \mathcal{S}\right\}, \quad J=\left\{\left(\begin{array}{ccc}
0 & m & 0 \\
0 & 0 & 0 \\
0 & 0 & 0
\end{array}\right) \mid m \in \mathcal{S}\right\} \text { and }
$$

$d\left(\begin{array}{lll}0 & x & y \\ 0 & 0 & 0 \\ 0 & z & 0\end{array}\right)=\left(\begin{array}{lll}0 & x & y \\ 0 & 0 & 0 \\ 0 & 0 & 0\end{array}\right)$. Then it can be seen easily that $\mathcal{N}$ is a left near-ring which is not 3-prime, $J$ a nonzero Jordan ideal of $\mathcal{N}$ and $d$ a nonzero derivation on $\mathcal{N}$ such that

(i) $d(j n) \in Z(\mathcal{N})$, for all $j \in J, n \in \mathcal{N}$,

(ii) $d(n \circ j)=[n, j]$, for all $j \in J, n \in \mathcal{N}$,

(iii) $d([n, j])=n \circ j$, for all $j \in J, n \in \mathcal{N}$,

(iv) $[d(n), j]=n \circ j$, for all $j \in J, n \in \mathcal{N}$,

(v) $d(n) \circ j=[n, j]$, for all $j \in J, n \in \mathcal{N}$.

However, $J$ is not commutative. 


\section{REFERENCES}

[1] N. Argaç and H. E. Bell, Some Results on Derivations in Near-rings, Near-rings and Near-fields, Stellenbosch, 1997, Kluwer Academic Publishers Dordrecht, 2001, 42-46.

[2] M. Ashraf and N. Rehman On commutativity of rings with derivations, Results Math. 42(1-2) (2002), 3-8.

[3] M. Ashraf and A. Shakir, On $(\sigma, \tau)$-derivations of prime near-rings-II, Sarajevo J. Math. 4(16) (2008), 2330.

[4] H. E. Bell and G. Mason, On derivations in near-rings in: Near-rings and Near-fields, (Tübingen, 1985), 31-35, North-Holland Math. Stud. 137, North-Holland, Amsterdam, 1987.

[5] H. E. Bell, On Derivations in Near-rings II, Kluwer Academic Publishers Netherlands, 1997, 191-197.

[6] A. Boua and L. Oukhtite, Derivations on prime near-rings, Int. J. Comput. Sci. Math. 4(2) (2011), 162-167.

[7] H. E. Bell, A. Boua and L. Oukhtite, On derivations of prime near-rings, Afr. Diaspora J. Math. 14(1) (2012), 65-72.

[8] A. Boua, L. Oukhtite and A. Raji, Jordan ideals and derivations in prime near-rings, Comment. Math. Univ. Carolin. 55(2) (2014), 131-139.

[9] A. Boua and A. A. M. Kamal, Lie ideals and Jordan ideals in 3-prime near-rings with derivations, JP J. Alg, Number Theory Appl. 55(2) (2014), 131-139.

[10] M. N. Daif and H. E. Bell, Remarks on derivations on semiprime rings, Internat. J. Math. and Math. Sci. 15 (1992), 205-206.

[11] A. A. M. Kamal and K. H. Al-Shaalan, Existence of derivations on near-rings, Math. Slovaca, 63(3) (2013), 431-448.

[12] G. Pilz, Near-rings. The Theory and its Applications, Second edition, North-Holland Mathematics Studies, 23. North-Holland Publishing Co. Amsterdam, 1983.

[13] X. K. Wang, Derivations in prime rings, Proc. Amer. Math. Soc. 121(2) (1994), 361-366.

1 "Department of Mathematics, Physics and Computer Science, Polydisciplinary Faculty, LSI, TAZA,

Sidi Mohammed Ben Abdellah University, Fez, Morocco

Email address: abdelkarimboua@yahoo.fr

${ }^{2}$ Department of Mathematics,

Aligarh Muslim University,

AligARH-202002

Email address: asma_ali2@rediffmail.com

Email address: inzamamulhuque057@gmail.com 